\title{
Efficacy and safety of anti-epidermal growth factor receptor therapy compared with anti-vascular endothelial growth factor therapy for metastatic colorectal cancer in first-line and second-line therapies: a meta-analysis
}

This article was published in the following Dove Press journal:

OncoTargets and Therapy

30 August 2016

Number of times this article has been viewed

\section{Hongchi Wang* \\ Bin $\mathrm{Ma}^{*}$ \\ Peng Gao \\ Yongxi Song \\ Qingzhou Xu \\ Yaoyuan Hu \\ Cong Zhang \\ Zhenning Wang}

Department of Surgical Oncology and General Surgery, The First Hospital of China Medical University, Shenyang,

People's Republic of China

*These authors contributed equally to this work
Correspondence: Zhenning Wang Department of Surgical Oncology and General Surgery, The First Hospital of China Medical University, North Nanjing Street 155, Shenyang I I000I,

People's Republic of China

Tel +86248328 3556

Fax +862422703578

Email josieon826@sina.cn
Aim: This study aimed to compare anti-epidermal growth factor receptor (anti-EGFR) therapy and anti-vascular endothelial growth factor therapy as first-line and second-line therapies in patients with KRAS exon 2 codon 12/13 wild-type (KRAS-WT) metastatic colorectal cancer (mCRC).

Methods: Major databases were systematically searched. The hazard ratio (HR), odds ratio (OR), and $95 \%$ confidence intervals ( $95 \%$ CIs) were used to estimate the effect measures. Review Manager software version 5.3 was used for statistical analysis.

Results: Seven trials including ten articles were eligible in the meta-analysis. The patients treated with anti-EGFR as first-line therapy showed a longer overall survival (OS) for KRAS-WT and all RAS wild-type (RAS-WT) $\mathrm{mCRC}$ (HR $=0.81,95 \% \mathrm{CI}: 0.72-0.92, P<0.01, \mathrm{n}=5$; HR $=0.78$, 95\% CI: $0.66-0.93, P<0.01, \mathrm{n}=3$, respectively). The objective response rate (ORR) was better with the anti-EGFR therapy for KRAS-WT and all RAS-WT mCRC (OR $=1.32,95 \% \mathrm{CI}$ : 1.11-1.56, $P<0.01, \mathrm{n}=5$; OR $=1.55,95 \% \mathrm{CI}: 1.21-2.00, P<0.01, \mathrm{n}=3$, respectively). There was no difference in progression-free survival (PFS) for KRAS-WT mCRC and all RAS-WT mCRC between the two groups $(\mathrm{HR}=1.00 ; 95 \% \mathrm{CI}: 0.92-1.09, P=0.99, \mathrm{n}=4 ; \mathrm{HR}=0.92,95 \%$ CI: $0.71-1.19, P=0.52, \mathrm{n}=3$, respectively). In addition, two trials provided data on the secondline therapy; there was no significant difference in OS and PFS for the second-line therapy, but a significant improvement in ORR was found in the anti-EGFR group (OR $=1.91,95 \% \mathrm{CI}$ : $1.16-3.16, P=0.01, \mathrm{n}=2)$. No difference in the conversion therapy ( $\mathrm{OR}=1.34 ; 95 \% \mathrm{CI}: 0.91-1.99$; $P=0.14, \mathrm{n}=4$ ) was observed between the two therapies.

Conclusion: Our results indicate that anti-EGFR therapy is superior to anti-vascular endothelial growth factor therapy for OS and ORR as a first-line therapy for KRAS-WT mCRC. In the second-line therapy, there was no significant difference in the survival outcomes on the basis of OS and PFS between the two groups. However, ORR improved significantly in the anti-EGFR group.

Keywords: colorectal cancer, anti-EGFR, anti-VEGF, chemotherapy, meta-analysis

\section{Introduction}

Colorectal cancer (CRC) is the third most common cancer among males and the second most common cancer among females, with an estimated death of 600,000 individuals in 2012 worldwide owing to advanced CRC. ${ }^{1}$ Although the surgical approach has ensured 
an improvement in the management of early and advanced $\mathrm{CRC}$, the prognosis of patients with metastatic CRC (mCRC) is still poor. ${ }^{2}$ The systemic therapeutic approach is the major choice of treatment for $\mathrm{mCRC}$. In the past decade, combination chemotherapies, including 5-fluorouracil, leucovorin, and oxaliplatin (FOLFOX) and fluorouracil, folinic acid, and irinotecan (FOLFIRI), have improved the survival outcomes significantly in patients with mCRC. ${ }^{3,4}$ More recently, studies have demonstrated that the addition of anti-epidermal growth factor receptor (anti-EGFR) or anti-vascular endothelial growth factor (anti-VEGF) to the conventional combination chemotherapy improved the survival outcomes compared with conventional combination chemotherapy. ${ }^{5-7}$ Therefore, antibodies to EGFR/VEGF in combination with FOLFOX or FOLFIRI have become the first- and second-line treatment options for patients with mCRC.

However, anti-EGFR therapy predicted a negative outcome for mCRC patients with mutations in codons 12 and 13 of KRAS exon 2 (up to $40 \%$ ). ${ }^{8}$ In addition, recent studies have indicated that less frequent mutations in RAS protooncogenes HRAS, KRAS, and NRAS, including mutations in exons 3 or 4 of KRAS and exons 2-4 of NRAS, predicted a lack of effectiveness of anti-EGFR therapies in mCRC patients. ${ }^{9,10}$ Therefore, the patients with such mutations who were excluded after RAS and KRAS analyses would have higher survival with anti-EGFR therapy combined with conventional combination therapy, compared with the conventional combination therapy alone. ${ }^{10,11}$ However, at present, there is no predictive biomarker for anti-VEGF therapy.

Recent studies have demonstrated conflicting results for the types of antibody (anti-EGFR or anti-VEGF) that provide better clinical efficacy for mCRC patients. ${ }^{12-15}$ A previous meta-analysis compared anti-EGFR and anti-VEGF therapies in the first-line setting on the basis of overall survival (OS), progression-free survival (PFS), and objective response rate (ORR). ${ }^{16}$ However, it did not compare the two therapies based on toxicity, second-line therapy, and conversion therapy. Therefore, we conducted this meta-analysis including randomized clinical trials and retrospective studies so as to give an overview of the results comparing anti-EGFR and anti-VEGF therapies as first- and second-line therapies based on survival outcomes, toxicity, and conversion rate in conversion therapy in patients with KRAS exon 2 wild-type (KRAS-WT) mCRC.

\section{Materials and methods}

\section{Search strategy}

This meta-analysis was conducted in accordance with Preferred Reporting Items for Systematic Reviews and
Meta-Analysis guidelines. ${ }^{17}$ We conducted a systematic literature search for journals published until January of 2016 using PubMed, EMBASE, and the Cochrane databases. We also searched for abstracts from the American Society of Clinical Oncology and European Society for Medical Oncology. The main search terms were "cancer of colon", "colorectal carcinoma”, "colorectal cancer", "cetuximab or panitumumab or anti-EGFR", and "bevacizumab or aflibercept or anti-VEGF". We also screened relevant abstracts, methods, and references of the retrieved articles. The search was limited to human studies, and the language of the searched publications was restricted to English.

\section{Inclusion criteria}

To ensure the accuracy and reliability of the analysis, the studies included in this meta-analysis met the following criteria: 1) studies involving patients diagnosed with KRAS-WT mCRC; 2) trials that compared anti-EGFR therapy and anti-VEGF therapy in association with combination chemotherapy as first-line or second-line chemotherapy for mCRC; and 3) studies that reported at least one of the following outcome measures: OS, PFS, ORR, toxicity, and conversion therapy.

\section{Exclusion criteria}

The exclusion criteria were as follows: 1) studies that evaluated fewer than 30 patients; 2) studies that lacked sufficient data necessary for analysis; 3 ) repeated studies that contained the same databases or patients; 4) letters, reviews, case reports, editorials, and expert opinions.

\section{Quality assessment}

Two independent reviewers (H. C. Wang and B. Ma) used the Jadad scale to assess the methodological quality of all eligible randomized controlled trials (RCTs) and the NewcastleOttawa Scale to evaluate the quality of the nonrandomized studies. ${ }^{18,19}$ Any disagreements between the two reviewers were resolved by a third reviewer.

\section{Data extraction}

Two researchers (H. C. Wang and B. Ma) independently extracted data from all eligible studies. The outputs for this meta-analysis included first author's name, year of publication, country of origin of the studies, study design, participants (number of patients and mean age), study regimen, Eastern Cooperative Oncology Group performance status score, ORR, OS, PFS, toxicity, and conversion therapy. Any disagreements were resolved by a third reviewer. 


\section{Statistical analysis}

The end points of this study were OS, PFS, ORR, toxicity, and conversion rate. ORR was defined as the sum of partial and complete response rates, according to the Response Evaluation Criteria in Solid Tumors. ${ }^{20}$ Toxicity was assessed using the National Cancer Institution Common Toxicity Criteria (version 2.0, http://ctep.cancer.gov). This metaanalysis was conducted using Review Manager software version 5.3 (The Nordic Cochrane Centre, The Cochrane Collaboration, Copenhagen, Denmark, 2014).

Time-to-event data (OS, PFS) analyses were performed via calculation of the hazard ratio (HR) and $95 \%$ confidence intervals (CIs). The pooled HR was calculated using the inverse-variance-weighted average of the individual studies. Dichotomous data (ORR, toxicity, and conversion rate) were represented by pooled estimates of odds ratios (ORs) and $95 \%$ CIs. A test with a $P$-value of $<0.05$ was considered statistically significant. The heterogeneity between studies was tested using the Cochran $Q$-test and $I$ index. $P$-values $<0.1$ and/ or $I^{2}>50 \%$ indicated significant heterogeneity. A randomeffects model was used in cases of significant heterogeneity. Otherwise, data were analyzed using a fixed-effects model. ${ }^{21}$ Publication bias was assessed by Begg's and Egger's tests based on the Stata software, version 12.0 (2011; Stata Corp, College Station, TX, USA). ${ }^{22,23}$ Subgroups analyses were performed considering the patient groups (KRAS-WT mCRC patients and all RAS-WT mCRC patients) and the chemotherapy backbones (FOLFOX and FOLFIRI).

\section{Results \\ Eligible studies}

We searched 3,090 studies (Figure 1), 61 of which were retrieved after the screening of titles and abstracts. Subsequently, 51 studies were excluded because of redundancy or the lack of an outcome of interest. Therefore, seven trials containing ten articles (the CALGB/SWOG80405 trial was found in meeting abstracts from four articles) were finally included in this meta-analysis. The baseline characteristics of the included trials are listed in Table 1. Eight eligible articles (the CALGB/SWOG80405 trial was found in meeting abstracts from four studies, all of which presented different outcomes) involving 1,117 patients in the anti-EGFR group and 1,193 patients in the anti-VEGF group compared two therapies used as the first-line setting (we accumulated the patients number from CALGB/SWOG80405 trial only once). ${ }^{12-15,24-27}$ Two articles (including one meeting abstract) containing 160 patients in the anti-EGFR group and 147 patients in the anti-VEGF group, respectively, were eligible for our study in the second-line setting..$^{28,29}$ All the RCTs had Jadad scores of $\geq 3$ and were considered to be high-quality studies. All the retrospective studies had Newcastle-Ottawa Scale score of 6 and were considered

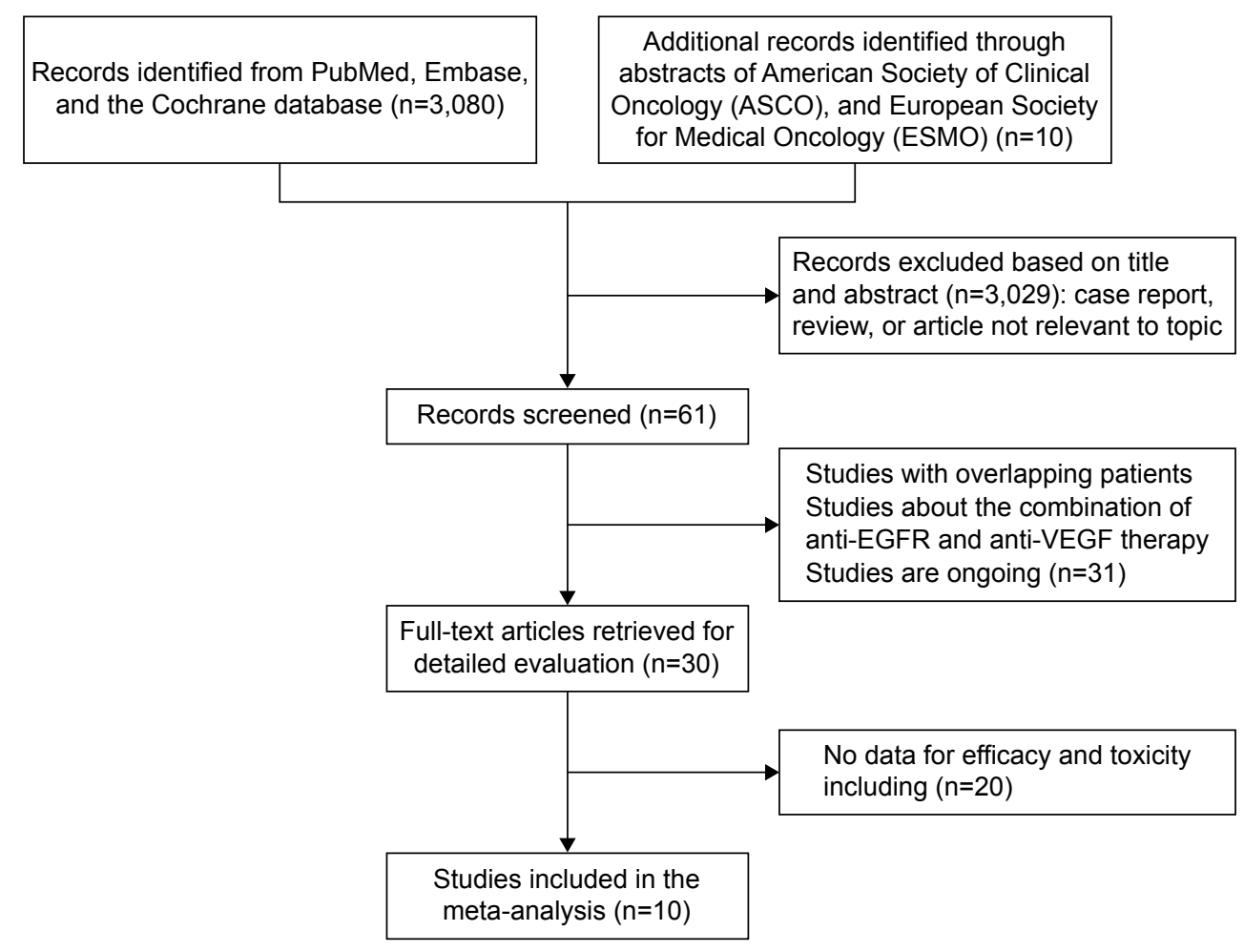

Figure I Flow chart of study selection. 
Table I Characteristics of studies included in this meta-analysis

\begin{tabular}{|c|c|c|c|c|c|c|c|c|}
\hline Study & Year & Country & $\begin{array}{l}\text { Study } \\
\text { design }\end{array}$ & $\begin{array}{l}\text { Treatment } \\
\text { groups }\end{array}$ & $\begin{array}{l}\text { No of } \\
\text { patients }\end{array}$ & Regimen & Age & PS \\
\hline \multirow[t]{2}{*}{ Heinemann et $\mathrm{al}^{12}$} & 2014 & Germany & Randomized & Group A & $A=297$ & A: FOLFIRI + cetuximab & $64(38-79)$ & $0-2$ \\
\hline & & & Phase III study & Group B & $B=295$ & B: FOLFIRI + bevacizumab & $65(27-76)$ & $0-2$ \\
\hline \multirow[t]{2}{*}{ Schwartzberg et al ${ }^{14}$} & 2014 & Spain & Randomized & Group A & $A=142$ & A: mFOLFOX6 + panitumumab & $63(23-82)$ & $0-1$ \\
\hline & & & Phase II study & Group B & $B=143$ & B: mFOLFOX6 + bevacizumab & $61(28-82)$ & $0-1$ \\
\hline CALGB/ & 2014 & USA & Randomized & Group A & $A=578$ & A: FOLFIRI/mFOLFOX6 + cetuximab & 59 (NA) & $0-1$ \\
\hline SWOG80405 $13,15,24,26$ & & & Phase III study & Group B & $B=559$ & B: FOLFIRI/mFOLFOX6 + bevacizumab & 59 (NA) & $0-1$ \\
\hline \multirow[t]{3}{*}{ Stremitzer et $\mathrm{al}^{25}$} & 2015 & Austria & Retrospective & Group A & $A=37$ & A: Fluoropyrimidine only/irinotecan/oxaliplatin + & $63(31-80)$ & NA \\
\hline & & & & Group B & $B=|0|$ & cetuximab & $63(31-80)$ & NA \\
\hline & & & & & & $\begin{array}{l}\text { B: irinotecan/irinotecan + oxaliplatin/oxaliplatin + } \\
\text { bevacizumab }\end{array}$ & & \\
\hline \multirow[t]{2}{*}{ Yang et $\mathrm{al}^{27}$} & 2014 & Taiwan & Retrospective & Group A & $A=63$ & A: irinotecan-based/oxaliplatin-based + cetuximab & NA & NA \\
\hline & & & & Group B & $B=95$ & B: irinotecan-based/oxaliplatin-based + bevacizumab & NA & NA \\
\hline \multirow[t]{2}{*}{ Heinemann et $\mathrm{al}^{29}$} & 2015 & Germany & Randomized & Group A & $A=69$ & A: FOLFIRI + cetuximab & NA & $0-1$ \\
\hline & & & Phase III study & Group B & $B=56$ & B: FOLFIRI + bevacizumab & NA & $0-1$ \\
\hline \multirow[t]{2}{*}{ Hecht et $\mathrm{a}^{28}$} & 2015 & USA & Randomized & Group A & $A=91$ & A: FOLFIRI + panitumumab & $60(27-84)$ & $0-1$ \\
\hline & & & Phase II study & Group B & $B=91$ & B: FOLFIRI + bevacizumab & $60(25-80)$ & $0-1$ \\
\hline
\end{tabular}

Abbreviations: Irinotecan-based, irinotecan-based combination therapy; Oxaliplatin-based, oxaliplatin-based combination therapy; NA, not applicable; FOLFIRI, fluorouracil, folinic acid, and irinotecan; FOLFOX, 5-fluorouracil, leucovorin, and oxaliplatin; PS, performance status; mFOLFOX, modified, 5-fluorouracil, leucovorin, oxaliplatin.

to be moderate-quality studies (Tables S1 and S2). In our meta-analysis, we included five abstracts because only these provided the results without a detailed description of materials and methods. Hence, we did not assess the quality of the five abstracts.

\section{Effectiveness of first-line therapy on the basis of OS, PFS, ORR, toxicity, and conversion therapy}

\section{Overall survival}

Five articles provided data on OS for KRAS-WT mCRC. . $11,15,25,27$ An improvement in OS was observed in the anti-EGFR group compared with the anti-VEGF group (HR $=0.81,95 \%$ CI: 0.72-0.92, $P<0.01, \mathrm{n}=5$ ) (Figure 2A). In addition, for all RAS-WT mCRC patients, the results of three studies on all RAS-WT mCRC corroborated the improvement in OS (HR $=0.78,95 \%$ CI: $0.66-0.93, P<0.01, \mathrm{n}=3$ ) (Figure 2B). ${ }^{12-14}$ Furthermore, for all RAS-WT mCRC patients, we performed subgroup analyses on the basis of FOLFOX and FOLFIRI. No significant difference was observed between the two therapies (FOLFOX subgroup, HR $=0.79,95 \% \mathrm{CI}$ : $0.60-1.04, P=0.09$; FOLFIRI subgroup, $\mathrm{HR}=0.86,95 \% \mathrm{CI}$ : $0.55-1.34, P=0.49$ ). ${ }^{12,14,24}$ (Figure 2C). Publication bias was assessed by Begg's and Egger's test. There was no evidence of publication bias for pooled analysis of OS ( $P_{\text {Begg }}=0.806$, $P_{\text {Egger }}=0.295$ ) (Figures S1 and S2).

\section{Progression-free survival}

Four articles on KRAS-WT mCRC ${ }^{12,14,15,27}$ and three studies on all RAS-WT mCRC ${ }^{12-14}$ provided data on PFS in the first-line therapy. There were no significant differences in PFS for KRAS-WT and all RAS-WT mCRC patients between the two therapies (HR $=1.00,95 \%$ CI: 0.92-1.09, $P=0.99$, $\mathrm{n}=4$; $\mathrm{HR}=0.92,95 \%$ CI: $0.71-1.19, P=0.52, \mathrm{n}=3$, respectively) (Figure $3 \mathrm{~A}$ and $\mathrm{B}$ ). In addition, for patients with all RAS-WT $\mathrm{mCRC}$, we observed no significant difference in PFS between the two therapies based on the FOLFOX or FOLFIRI regimen $(\mathrm{HR}=0.87,95 \%$ CI: $0.52-1.46, P=0.60 ; \mathrm{HR}=0.98,95 \%$ CI: $0.80-1.19, P=0.83$, respectively) ${ }^{12,14,24}$ (Figure $3 \mathrm{C}$ ).

\section{Toxicity}

We evaluated the differences in Grade 3 toxicity or lower between the anti-EGFR and anti-VEGF groups. Three articles presented data on toxicity, and these studies are listed in Table $2 .^{12,14,27}$ The pooling analysis of relevant studies indicated a significant increase in the occurrence of skin disorders, hypomagnesemia, and hypokalemia in the anti-EGFR group compared with the anti-VEGF group (skin disorders: $\mathrm{OR}=20.35,95 \% \mathrm{CI}$ : 9.82-42.17, $P<0.01$, $\mathrm{n}=2$; hypomagnesemia: $\mathrm{OR}=9.35,95 \%$ CI: $2.52-34.69$, $P<0.01, \mathrm{n}=2$; hypokalemia: $\mathrm{OR}=2.43$; $95 \%$ CI: $1.33-4.44$, $P<0.01, \mathrm{n}=2)$. By contrast, there was a significant increase in hypertension in the anti-VEGF group (OR $=0.12,95 \%$ CI: $0.02-0.62, P=0.01, \mathrm{n}=2)$. No significant differences in fatigue, stomatitis, dehydration, decreased appetite, nausea, and hypocalcemia were found between the two groups.

\section{Objective response rate}

For patients with KRAS-WT mCRC, data on ORR were available from five trials. ${ }^{12,14,15,25,27}$ A total of 709 patients 


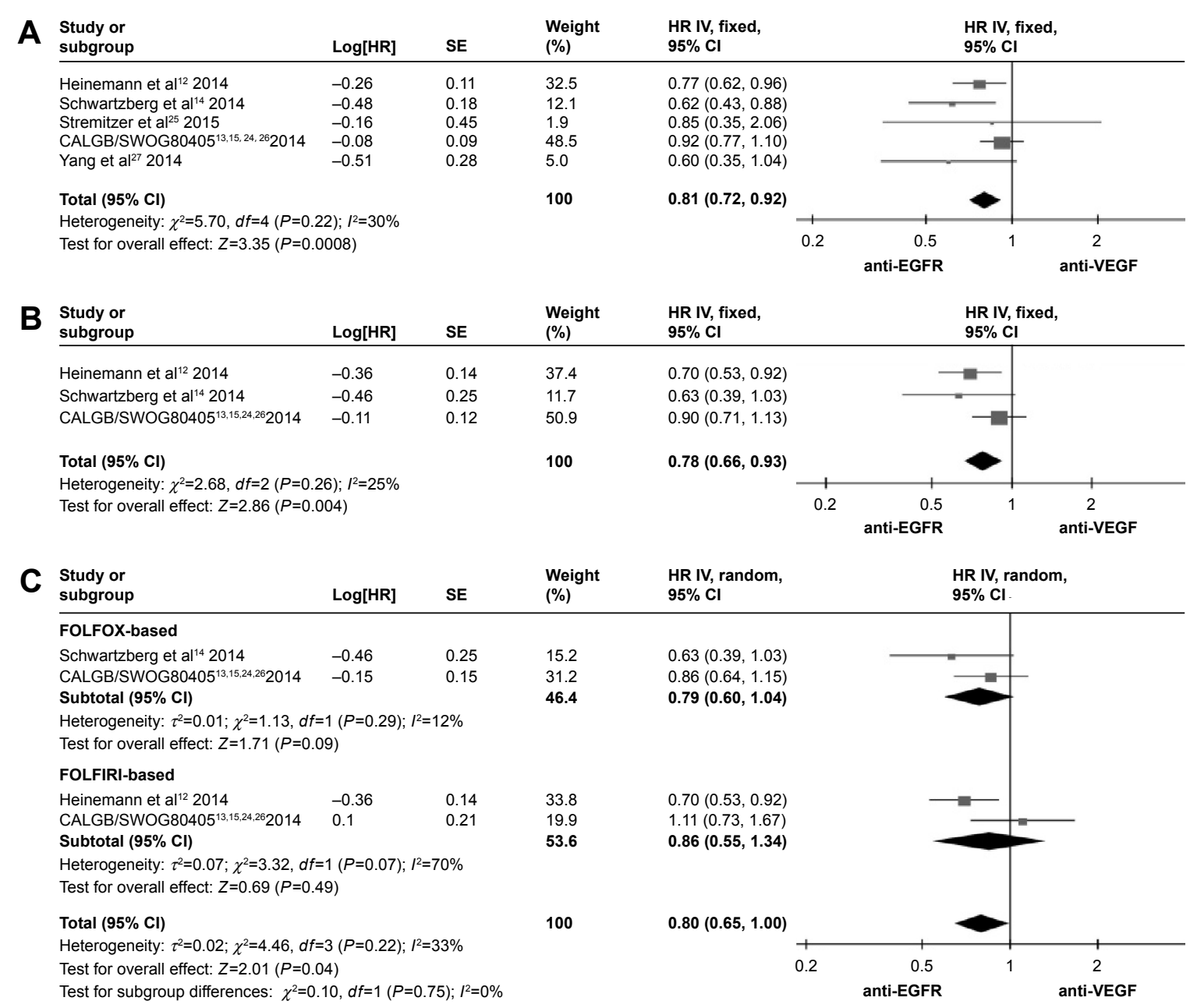

Figure 2 Forest plots and subgroup analysis.

Notes: (A) Forest plot of HR for OS in KRAS-WT mCRC patients; (B) Forest plot of HR for OS in all RAS-WT mCRC patients. (C) Subgroup analysis for OS based on FOLFIRI and FOLFOX.

Abbreviations: HR, hazard ratio; OS, overall survival; Cl, confidence interval; FOLFIRI, fluorouracil, folinic acid, and irinotecan; FOLFOX, 5-fluorouracil, leucovorin, and oxaliplatin; WT, wild type; mCRC, metastatic colorectal cancer; anti-EGFR, anti-epidermal growth factor receptor; anti-VEGF, anti-vascular endothelial growth factor; $\mathrm{SE}$, standard error; $d f$, degrees of freedom.

$(709 / 1,106,64 \%)$ in the anti-EGFR group and 689 patients $(689 / 1,169,59 \%)$ in the anti-VEGF group achieved an objective response. A significant improvement in ORR was observed in the anti-EGFR group $(\mathrm{OR}=1.32,95 \% \mathrm{CI}: 1.11-1.56, P<0.01$, $\mathrm{n}=5$ ) (Figure 4A). In addition, three articles provided data on all RAS-WT mCRC. ${ }^{12-14}$ A similar improvement in ORR was observed in all RAS-WT mCRC patients $(\mathrm{OR}=1.55,95 \% \mathrm{CI}$ : $1.21-2.00, P<0.01, \mathrm{n}=3$ ) (Figure 4B).

\section{Conversion therapy}

Anti-EGFR and anti-VEGF therapies can improve metastasectomy outcomes by converting unresectable metastatic disease into resectable disease. Four studies presented data on conversion therapy. ${ }^{12,14,26,27}$ A total of 183 patients $(183 / 1,080)$ underwent surgical resection in the anti-EGFR group $(17 \%)$ whereas 150 patients $(150 / 1,092)$ underwent surgical resection in the anti-VEGF group (14\%).
There was no significant difference in this outcome between the two therapies $(\mathrm{OR}=1.34,95 \% \mathrm{CI}$ : 0.91-1.99, $P=0.14$, $\mathrm{n}=4$ ) (Figure 4C). However, we found a clear tendency for conversion therapy in the anti-EGFR therapy compared with the anti-VEGF therapy.

\section{Effectiveness of second-line therapy on the basis of OS, PFS, and ORR}

Two articles (including one meeting abstract) provided data on the comparison between anti-EGFR and anti-VEGF therapies in combination with FOLFIRI for KRAS-WT $\mathrm{mCRC}$ when the disease progressed during oxaliplatin-based chemotherapy. ${ }^{28,29}$ There was no significant difference in OS (HR $=1.17,95 \%$ CI: 0.88-1.56, $P=0.29, \mathrm{n}=2$ ) (Figure 5A) and PFS $(\mathrm{HR}=1.12,95 \% \mathrm{CI}: 0.88-1.43, P=0.36, \mathrm{n}=2)$ (Figure 5B) between the two therapies. However, there was a significant improvement in ORR in the anti-EGFR group 


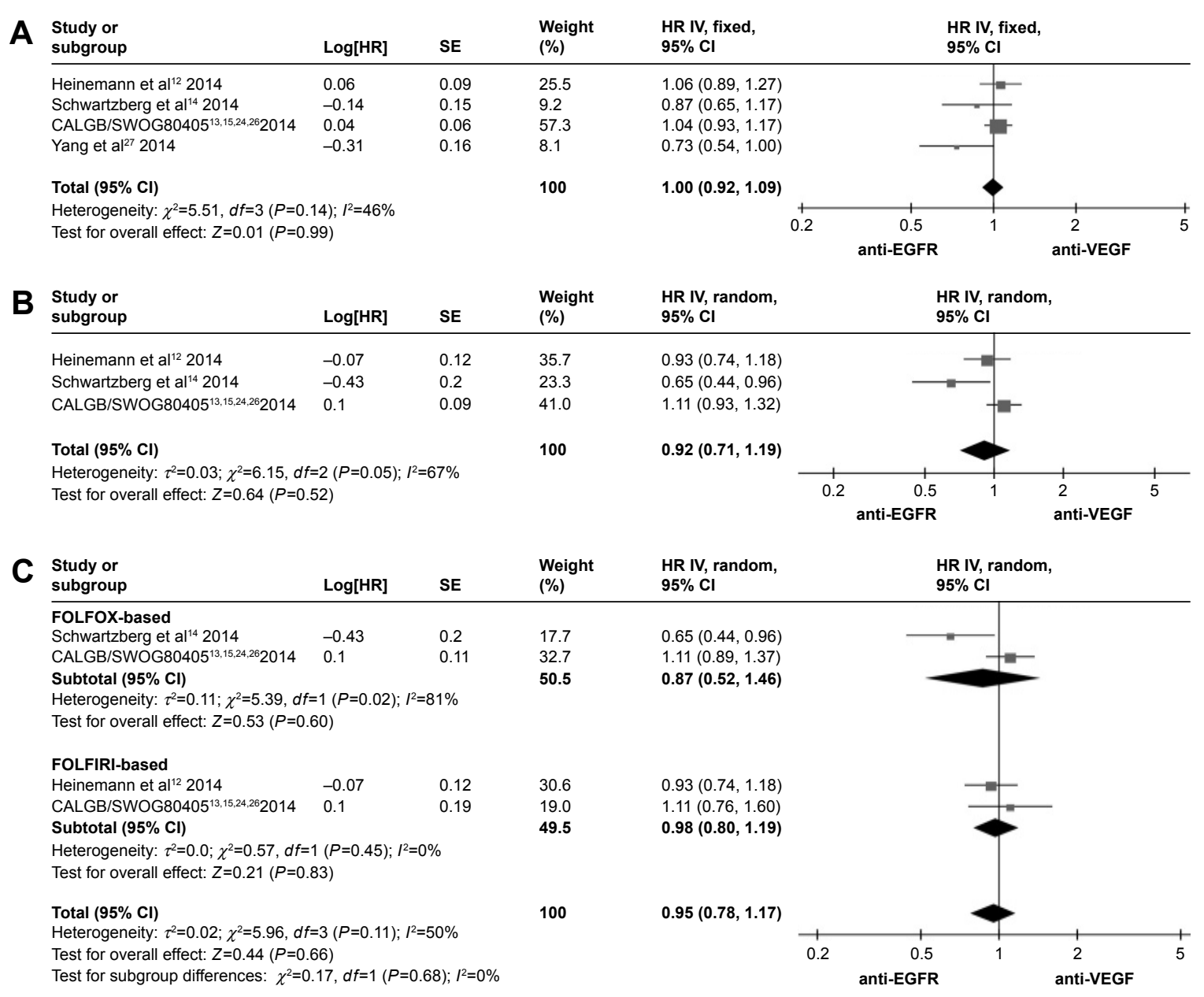

Figure 3 Forest plots.

Notes: (A) HR for PFS in KRAS-WT mCRC patients. (B) HR for PFS in all RAS-WT mCRC patients. (C) Subgroup analysis for PFS based on FOLFIRI and FOLFOX. Abbreviations: HR, hazard ratio; $\mathrm{Cl}$, confidence interval; PFS, progression-free survival; FOLFIRI, fluorouracil, folinic acid, and irinotecan; FOLFOX, 5-fluorouracil, leucovorin, and oxaliplatin; WT, wild type; mCRC, metastatic colorectal cancer; anti-EGFR, anti-epidermal growth factor receptor; anti-VEGF, anti-vascular endothelial growth factor; SE, standard error; $d f$, degrees of freedom.

when it was used as the second-line therapy $(\mathrm{OR}=1.91,95 \%$ CI: $1.16-3.16, P=0.01, \mathrm{n}=2$ ) (Figure 5C).

\section{Discussion}

In recent years, molecularly targeted therapies, including anti-EGFR and anti-VEGF therapies, have been applied in the treatment of mCRC. Studies have reported that the inclusion of anti-EGFR or anti-VEGF agents to combination chemotherapy improved the survival outcomes in mCRC. $5,30,31$ A recent review described the trials that compared the clinical efficacy and toxicity of anti-EGFR therapy and anti-VEGF therapy as a first-line therapy for KRAS-WT mCRC patients. ${ }^{32}$

Table 2 Toxicities (Grade 3 or lower) comparison between anti-EGFR therapy and anti-VEGF therapy

\begin{tabular}{|c|c|c|c|c|c|c|}
\hline Toxicity & Trials & $\begin{array}{l}\text { Anti-EGFR } \\
\text { based (n) }\end{array}$ & $\begin{array}{l}\text { Anti-VEGF } \\
\text { based (n) }\end{array}$ & $\begin{array}{l}\text { Heterogeneity } \\
P \text {-value }\left(I^{2}, \%\right)\end{array}$ & OR (95\% CI) & P-value \\
\hline Skin disorders & 2 & 121 & 8 & $0.46(0.0)$ & $20.35(9.82,42.17)$ & $<0.01$ \\
\hline Hypomagnesemia & 2 & 22 & 2 & $0.42(0.0)$ & 9.35 (2.52, 34.69) & $<0.01$ \\
\hline Fatigue & 2 & 17 & 16 & $0.32(0.0)$ & $1.07(0.52,2.17)$ & 0.86 \\
\hline Stomatitis & 2 & 18 & 13 & $0.07(7 I)$ & $2.06(0.27,15.82)$ & 0.49 \\
\hline Hypokalemia & 2 & 37 & 16 & $0.86(0.0)$ & $2.43(1.33,4.44)$ & $<0.01$ \\
\hline Dehydration & 2 & 8 & 5 & $0.07(7 I)$ & $1.61(0.13,19.83)$ & 0.71 \\
\hline Hypocalcemia & 2 & 7 & 7 & $0.23(31)$ & $0.99(0.36,2.76)$ & 0.99 \\
\hline Decreased appetite & 2 & II & 5 & $0.37(0.0)$ & $2.23(0.77,6.46)$ & 0.14 \\
\hline Nausea & 3 & 18 & 22 & $0.54(0)$ & $0.93(0.49,1.75)$ & 0.81 \\
\hline Hypertension & 2 & I & 14 & $0.23(31)$ & $0.12(0.02,0.62)$ & 0.01 \\
\hline
\end{tabular}

Abbreviations: anti-EGFR, anti-epidermal growth factor receptor; anti-VEGF, anti-vascular endothelial growth factor; OR, odds ratio; Cl, confidence interval. 


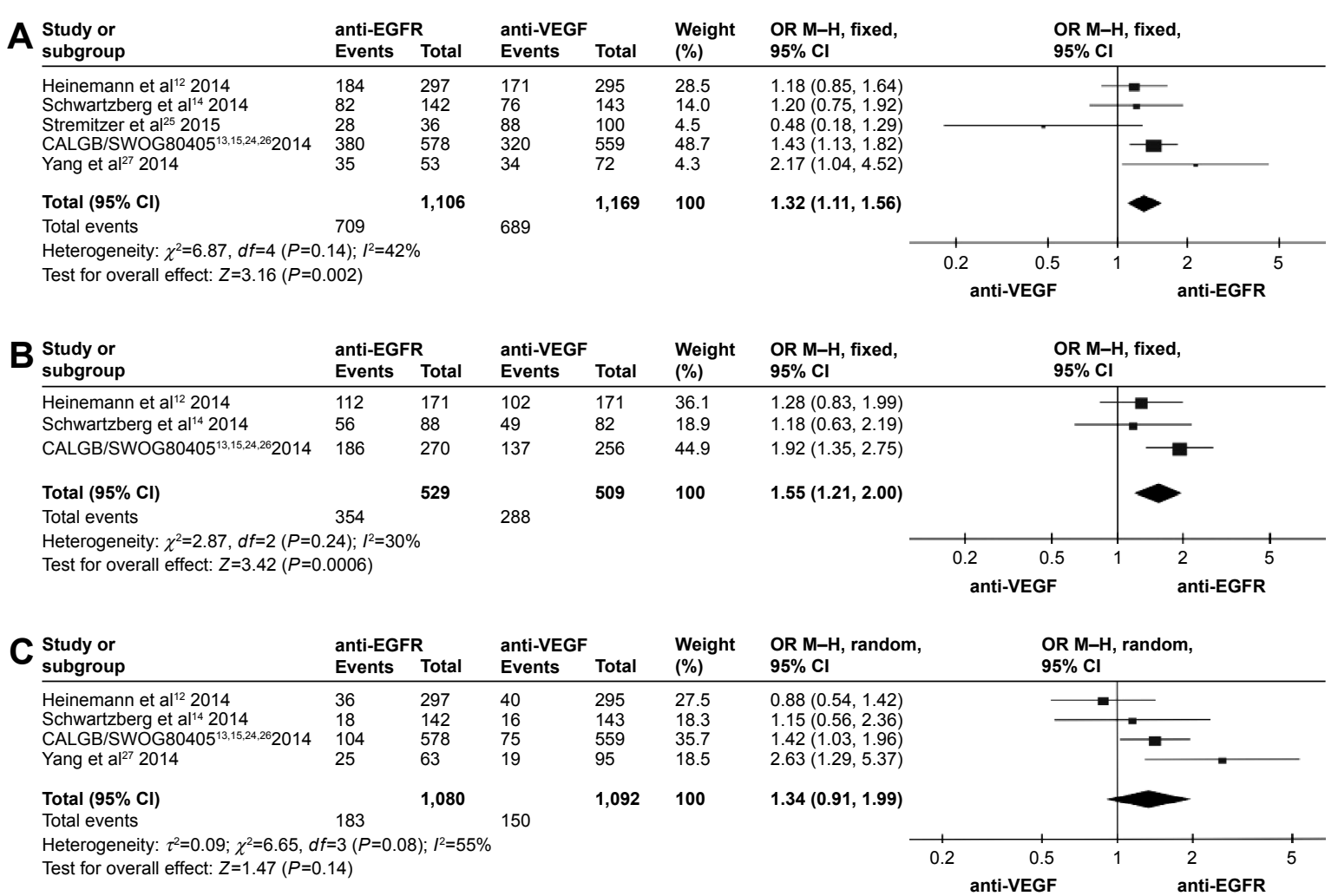

Figure 4 (A) OR for ORR in KRAS-WT mCRC patients, (B) OR for ORR in all RAS-WT mCRC patients, (C) OR for conversion therapy in KRAS-WT mCRC patients. Abbreviations: OR, odds ratio; ORR, objective response rate; $\mathrm{Cl}$, confidence interval; FOLFIRI, fluorouracil, folinic acid, and irinotecan; FOLFOX, 5-fluorouracil, leucovorin, and oxaliplatin; WT, wild type; mCRC, metastatic colorectal cancer; anti-EGFR, anti-epidermal growth factor receptor; anti-VEGF, anti-vascular endothelial growth factor; $\mathrm{SE}$, standard error; $d f$, degrees of freedom.

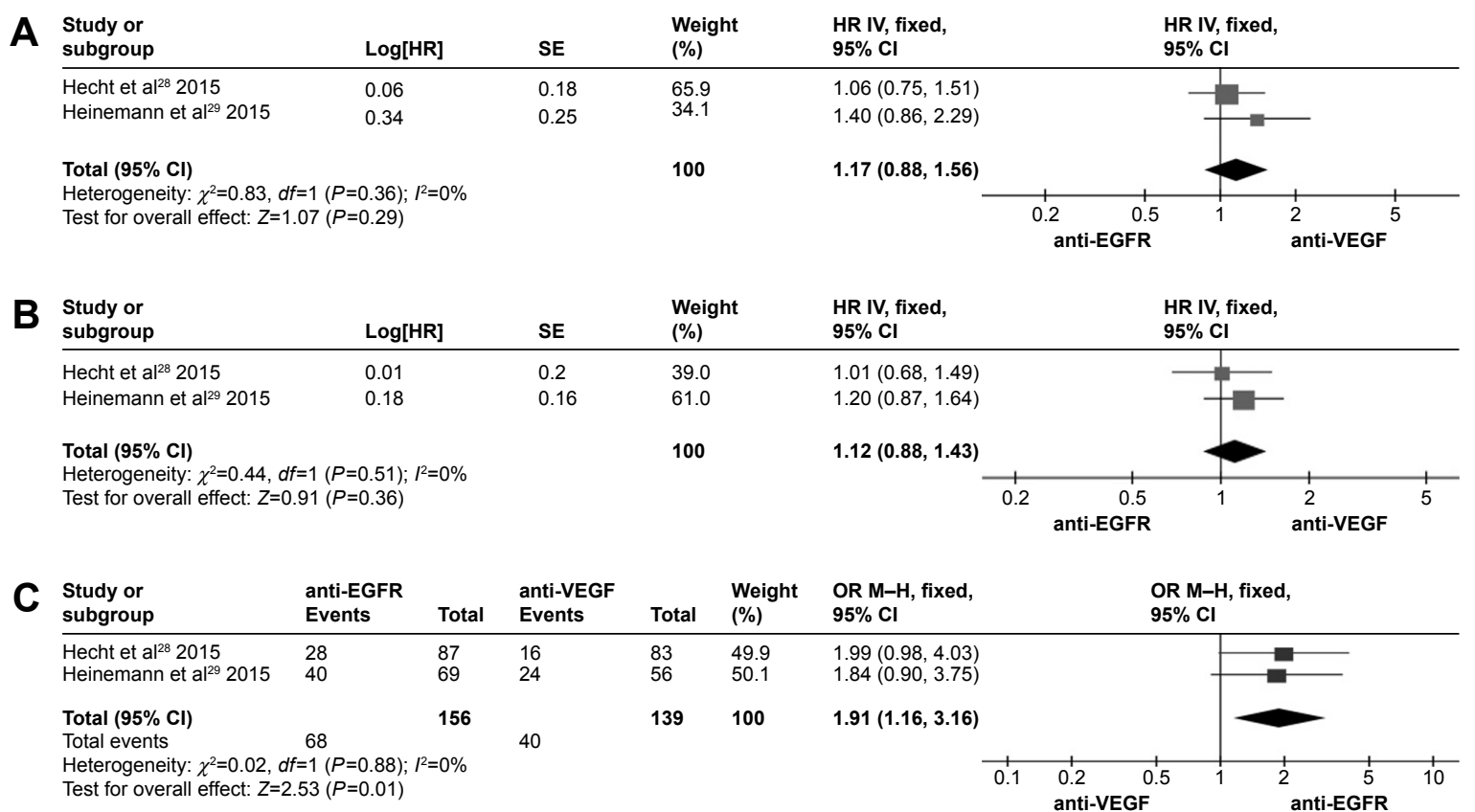

Figure 5 (A) HR for OS in KRAS-WT mCRC patients as a second-line therapy, (B) HR for PFS in KRAS-WT mCRC patients as a second-line therapy, (C) OR for ORR in KRAS-WT mCRC patients as a second-line therapy.

Abbreviations: HR, hazard ratio; OS, overall survival; PFS, progression-free survival; OR, Odds ratio; ORR, objective response rate; Cl, confidence interval; FOLFIRI, fluorouracil, folinic acid, and irinotecan; FOLFOX, 5-fluorouracil, leucovorin, and oxaliplatin; WT, wild type; mCRC, metastatic colorectal cancer; anti-EGFR, anti-epidermal growth factor receptor; anti-VEGF, anti-vascular endothelial growth factor; SE, standard error; $d f$, degrees of freedom. 
However, conflicting results between these trials were observed, and the optimal targeted combination chemotherapy as first-line and second-line treatments for mCRC patients is still unclear. A previous meta-analysis that evaluated the FIRE-3, PEAK, and CALGB/SWOG80405 trials concluded that ORR and OS were superior in the anti-EGFR therapy compared with anti-VEGF therapy for KRAS-WT mCRC, particularly in all RAS-WT mCRC patients; therefore, anti-EGFR therapy may be an alternative to anti-VEGF therapy as the initial treatment for mCRC. ${ }^{16}$ However, the results of previous meta-analyses were restricted by the limited number of eligible studies, and it did not provide data on toxicity, conversion therapy, and second-line therapy between the two groups. Our meta-analysis added retrospective studies and provided a more comprehensive comparison between anti-EGFR and anti-VEGF therapies for KRAS-WT mCRC patients in the first-line therapy on the basis of OS, PFS, ORR, conversion therapy, and toxicity and in the second-line therapy on the basis of OS, PFS, and ORR. In our meta-analysis, eight eligible studies provided data on survival outcomes in the first-line therapy for mCRC patients, and three of the included studies were RCTs. These RCTs (CALGB 80405 trial was found in meeting abstracts from four studies) have reported conflicting results on OS, PFS, and ORR in KRAS-WT mCRC patients. ${ }^{12,14,15}$ The analysis of the FIRE-3 and PEAK trials indicated a significant improvement in OS in the anti-EGFR group. By contrast, the randomized Phase III CALGB 80405 study found no significant difference in OS in KRAS-WT mCRC between the two therapies. In addition, the analysis of these RCTs indicated no significant difference in PFS between the two therapies. Similarly, the analysis of the FIRE-3 and PEAK trials indicated no significant difference in ORR between the two therapies. However, the CALGB 80405 study found a significant improvement in ORR in anti-EGFR therapy. Our meta-analysis indicated a significant improvement in ORR and OS in anti-EGFR therapy for KRAS-WT and all RAS-WT mCRC patients but no significant difference in PFS between the two therapies as the initial treatment for mCRC patients. The survival outcomes based on OS, PFS, ORR of our meta-analysis were consistent with the previous meta-analysis. These results allowed us to hypothesize the presence of a survival benefit in the anti-EGFR group compared with the anti-VEGF group as a first-line therapy for KRAS-WT mCRC patients.

It was a somewhat puzzling result that there was a significant difference in OS but no apparent difference in PFS between two groups in our meta-analysis and other eligible studies. There were some explanations. One explanation for this result is that a recent independent radiological review study demonstrated that higher early tumor shrinkage and higher depth of response were associated with improved OS. These effects were more pronounced in the anti-EGFR therapy compared with the anti-VEGF therapy, and this might explain the significant OS benefit of anti-EGFR associated with the combination chemotherapy. ${ }^{33}$ Another explanation is the effect of the second-line treatment and other therapies. A study on subsequent therapies for mCRC patients suggested that anti-EGFR therapy followed by the second-line anti-VEGF therapy could result in higher survival compared with the second-line anti-VEGF therapy followed by antiEGFR therapy. ${ }^{34}$ The upregulation of VEGF associated with the resistance to cetuximab has been reported in experimental models and favors the use of the second-line anti-VEGF therapy after the first-line anti-EGFR therapy. ${ }^{35,36}$ In addition, an eligible study in our meta-analysis found that the PFS benefit of anti-EGFR therapy relative to anti-VEGF therapy was only observed in patients with measurable tumor who achieved objective tumor response to biochemotherapy. ${ }^{27}$ This result indicated that only some subpopulations could achieve a PFS benefit by the anti-EGFR therapy. However, the CALGB 80405 trial found no significant difference in OS. This result may be because $73.4 \%$ of the patients received first-line FOLFOX, which might not be the best chemotherapy drug in combination with anti-EGFR therapy. ${ }^{37}$ Therefore, there was a selection bias in the chemotherapy backbones.

The prognosis of patients with $\mathrm{mCRC}$ is very poor, and a systemic therapeutic approach is the major choice of treatment for these patients. However, tumors of some patients might revert back to a resectable state in response to the conversion chemotherapy. ${ }^{2}$ Our meta-analysis evaluated the difference in conversion therapy between anti-EGFR therapy and anti-VEGF therapy. We found a clear trend toward the use of anti-EGFR therapy despite the lack of a significant difference between the two groups. The response rate and early tumor shrinkage are short-term indicators in conversion therapy..$^{38}$ Our meta-analysis indicated a significant improvement in ORR in the anti-EGFR therapy. In addition, the FIRE-3 trial demonstrated that, for the population evaluated, anti-EGFR therapy improved ORR compared with the anti-VEGF therapy. ${ }^{33}$ These results may indicate that the improved conversion therapy favors anti-EGFR therapy compared with anti-VEGF therapy.

We highlight the importance of toxicity of these two therapies in the first-line setting. A previous study has found that anti-EGFR therapy can cause infusion-related reactions 
and skin alterations. ${ }^{39}$ Moreover, anti-VEGF therapy can cause arterial hypertension, venous and arterial thromboembolic events, and gastrointestinal perforations. ${ }^{40}$ Our metaanalysis indicated that, in the anti-EGFR group, there was a significant increase in Grade 3 toxicity or lower, including skin disorders, hypomagnesemia, and hypokalemia, whereas the patients in the anti-VEGF group experienced hypertension more often. Therefore, we should evaluate the toxicity profiles and patient preferences when choosing between these two therapies because toxicity may have an adverse influence on the physical and psychological status of the patients.

The inclusion of RAS testing of/in all patients with mCRC could identify those who are potentially sensitive to anti-EGFR therapy. In our study, the exclusion of the patients with mutant RAS mCRC increased the OS in the anti-EGFR therapy compared with the anti-VEGF therapy. In addition, trials demonstrated that mutations in $R A S$ genes, in addition to mutations in KRAS exon 2, were negative predictive factors in anti-EGFR therapy. ${ }^{41,42}$ Furthermore, retrospective analyses suggested that mutations in the $B R A F$ gene might predict the efficacy of anti-EGFR therapy in mCRC. ${ }^{43,44}$ It is important to extend RAS analysis and carry out BRAF analysis to identify differences in survival outcomes in antiEGFR therapy. The merging of the data from the FIRE-3, PEAK, and CALGB/SWOG 80405 studies revealed that the chemotherapy backbone for all RAS-WT mCRC patients did not yield significant differences in OS and PFS between the two therapies in combination with FOLFOX or FOLFIRI regimen. However, our meta-analysis did not provide sufficient data and only made an indirect comparison regarding this issue. A previous meta-analysis reported the superior efficacy of anti-EGFR therapy when used in combination with an irinotecan-based regimen compared with an oxaliplatin-based chemotherapy. ${ }^{45}$ However, the PRIME trial provided evidence of improvement in survival outcomes for KRAS-WT mCRC patients when the anti-EGFR agent was combined with FOLFOX chemotherapy. ${ }^{30}$ These results indicate that these studies provided no definitively preferred backbone of chemotherapy and that additional studies are needed to elucidate this association. ${ }^{46}$

Trials have concluded that anti-EGFR and anti-VEGF therapies together with combination chemotherapy can improve survival outcomes in the second-line therapy for mCRC compared with combination chemotherapy alone. ${ }^{47,48}$ Our meta-analysis compared two therapies in the second-line setting and indicated that anti-EGFR therapy significantly improved ORR, whereas no significant differences in OS and PFS were observed between these two therapies. However, we identified a trend toward improvement in the OS in antiVEGF therapy. There are some explanations for this result. On one hand, more patients on anti-VEGF therapy received subsequent-line therapies. On the other hand, the SPIRITT trial reported that more patients on anti-EGFR therapy were older, had colon cancer, and two or more organs presented metastatic disease..$^{28}$ Our meta-analysis suggested that survival outcomes in anti-EGFR and anti-VEGF therapies were similar as second-line therapies. Therefore, the development of accurate biomarkers and further toxicological analyses in the second-line setting may help to identify the best therapy for individual patients.

Our meta-analysis had some limitations. First, the quality of the trials affected the results, and two of the eligible studies in our meta-analysis were not RCTs. Second, the sample size of the eligible studies was relatively small, leading to a relatively low statistical power. Third, our study included the CALGB/SWOG 80405 trial - presented in the abstracts from the American Society of Clinical Oncology and European Society for Medical Oncology conferences - and it did not provide sufficient data (such as toxicity) for analysis. Fourth, one of the included studies evaluated patients with mutations in KRAS codons 12 and 13 in anti-VEGF therapy, which might have caused bias. ${ }^{27}$ Fifth, we could not control or avoid the occurrence of relevant bias (ie, age, sex, and treatment regimen) in the pooling analysis. Sixth, heterogeneity was observed across the studies, and we adjusted for this factor by using a random-effects model to make our results statistically credible.

\section{Conclusion}

Our results indicate that anti-EGFR therapy improved OS and ORR and caused the toxicity expected compared with anti-VEGF therapy as a first-line therapy for KRAS-WT and all RAS-WT mCRC. Furthermore, we found a clear tendency for conversion therapy in the anti-EGFR group. There was a significant improvement in ORR in the second-line setting in the anti-EGFR group. Therefore, more high-quality and welldesigned studies are needed to provide further evidence.

\section{Acknowledgments}

This work was supported by Natural Science Foundation of Liaoning Province (No 2014029201), Program of Education Department of Liaoning Province (L2014307), the Key Laboratory Programme of Education Department of Liaoning Province (LZ2015076), and Scientific Programme of Science and Technology Department of Liaoning Province (2015225002). 


\section{Disclosure}

The authors report no conflicts of interest in this work.

\section{References}

1. Torre LA, Bray F, Siegel RL, Ferlay J, Lortet TJ, Jemal A. Global cancer statistics, 2012. CA Cancer J Clin. 2015;65(2):87-108.

2. Glimelius B, Cavalli-Bjorkman N. Metastatic colorectal cancer: current treatment and future options for improved survival. Medical approach present status. Scand J Gastroenterol. 2012;47(3):296-314.

3. Colucci G, Gebbia V, Paoletti G, et al. Phase III randomized trial of FOLFIRI versus FOLFOX4 in the treatment of advanced colorectal cancer: a multicenter study of the Gruppo Oncologico Dell'Italia Meridionale. J Clin Oncol. 2005;23(22):4866-4875.

4. Poston GJ, Figueras J, Giuliante F, et al. Urgent need for a new staging system in advanced colorectal cancer. J Clin Oncol. 2008;26(29): $4828-4833$.

5. Hurwitz H, Fehrenbacher L, Novotny W, et al. Bevacizumab plus irinotecan, fluorouracil, and leucovorin for metastatic colorectal cancer. N Engl J Med. 2004;350(23):2335-2342.

6. Van Cutsem E, Kohne CH, Hitre E, et al. Cetuximab and chemotherapy as initial treatment for metastatic colorectal cancer. $N$ Engl $J$ Med. 2009;360(14):1408-1417.

7. Van Cutsem E, Kohne CH, Lang I, et al. Cetuximab plus irinotecan, fluorouracil, and leucovorin as first-line treatment for metastatic colorectal cancer: updated analysis of overall survival according to tumor KRAS and BRAF mutation status. J Clin Oncol. 2011;29(15):2011-2019.

8. Amado RG, Wolf M, Peeters M, et al. Wild-type KRAS is required for panitumumab efficacy in patients with metastatic colorectal cancer. J Clin Oncol. 2008;26(10):1626-1634.

9. Kaczirek K, Ciuleanu TE, Vrbanec D, et al. FOLFOX4 plus cetuximab for patients with previously untreated metastatic colorectal cancer according to tumor RAS and BRAF mutation status: updated analysis of the CECOG/ CORE 1.2.002 study. Clin Colorectal Cancer. 2015;14(2):91-98.

10. Douillard JY, Oliner KS, Siena S, et al. Panitumumab-FOLFOX4 treatment and RAS mutations in colorectal cancer. N Engl J Med. 2013; 369(11):1023-1034.

11. Douillard JY, Siena S, Cassidy J, et al. Randomized, Phase III trial of panitumumab with infusional fluorouracil, leucovorin, and oxaliplatin (FOLFOX4) versus FOLFOX4 alone as first-line treatment in patients with previously untreated metastatic colorectal cancer: the PRIME study. J Clin Oncol. 2010;28(31):4697-4705.

12. Heinemann V, von Weikersthal LF, Decker T, et al. FOLFIRI plus cetuximab versus FOLFIRI plus bevacizumab as first-line treatment for patients with metastatic colorectal cancer (FIRE-3): a randomised, open-label, phase 3 trial. Lancet Oncol. 2014;15(10):1065-1075.

13. Lenz H, Niedzwiecki D, Innocenti F, et al. CALGB/SWOG 80405: Phase III trial of irinotecan/5-FU/leucovorin (FOLFIRI) or oxaliplatin/5-FU/leucovorin (mFOLFOX6) with bevacizumab (BV) or cetuximab (CET) for patients (pts) with expanded RAS analyses untreated metastatic adenocarcinoma of the colon or rectum (mCRC). J Clin Oncol. 2014;32:(Suppl; abstract LBA3).

14. Schwartzberg LS, Rivera F, Karthaus M, et al. PEAK: a randomized, multicenter Phase II study of panitumumab plus modified fluorouracil, leucovorin, and oxaliplatin (mFOLFOX6) or bevacizumab plus mFOLFOX6 in patients with previously untreated, unresectable, wild-type KRAS exon 2 metastatic colorectal cancer. J Clin Oncol. 2014;32(21):2240-2247.

15. Venook AP, Donna Niedzwiecki, Lenz H, et al. CALGB/SWOG80405: Phase III trial of irinotecan/5-FU/leucovorin (FOLFIRI) or oxaliplatin/5-FU/leucovorin (mFOLFOX6) with bevacizumab (BV) or cetuximab (CET) for patients (pts) with KRAS wild-type (wt) untreated metastatic adenocarcinoma of the colon or rectum (MCRC). J Clin Oncol. 2014;32(5s):(Suppl; abstract LBA3).

16. Khattak MA, Martin H, Davidson A, Phillips M. Role of first-line antiepidermal growth factor receptor therapy compared with anti-vascular endothelial growth factor therapy in advanced colorectal cancer: a meta-analysis of randomized clinical trials. Clin Colorectal Cancer. 2015;14(2):81-90.
17. Panic N, Leoncini E, de Belvis G, Ricciardi W, Boccia S. Evaluation of the endorsement of the preferred reporting items for systematic reviews and meta-analysis (PRISMA) statement on the quality of published systematic review and meta-analyses. PloS One. 2013;8(12):e83138.

18. Moher D, Pham B, Jones A, et al. Does quality of reports of randomised trials affect estimates of intervention efficacy reported in meta-analyses? Lancet. 1998;352(9128):609-613.

19. Stang A. Critical evaluation of the Newcastle-Ottawa scale for the assessment of the quality of nonrandomized studies in meta-analyses. Eur J Epidemiol. 2010;25(9):603-605.

20. Therasse P, Arbuck SG, Eisenhauer EA, et al. New guidelines to evaluate the response to treatment in solid tumors. European Organization for Research and Treatment of Cancer, National Cancer Institute of the United States, National Cancer Institute of Canada. J Natl Cancer Inst. 2000;92(3):205-216.

21. Schmidt FL, Oh IS, Hayes TL. Fixed- versus random-effects models in meta-analysis: model properties and an empirical comparison of differences in results. Br J Math Stat Psychol. 2009;62(Pt 1):97-128.

22. Egger M, Davey Smith G, Schneider M, Minder C. Bias in meta-analysis detected by a simple, graphical test. BMJ. 1997;315(7109):629-634.

23. Begg CB, Mazumder M. Operating characteristics of a rank correlation test for publication bias. Biometrics. 1994;50(4):1088-1101.

24. Calson RH. Matastatic colorectal cancer: antibody regimens found equivalent. Oncol Times. 2014;36(13):28-29.

25. Stremitzer S, Stift J, Singh J, et al. Histological response, pattern of tumor destruction and clinical outcome after neoadjuvant chemotherapy including bevacizumab or cetuximab in patients undergoing liver resection for colorectal liver metastases. Eur J Surg Oncol. 2015; 41(7):868-874.

26. Venook A, Niedzwiecki D, Lenz H, et al. LBA10CALGB/SWOG 80405: analysis of patients undergoing surgery as part of treatment strategy. Ann Oncol. 2014;25(Suppl 4):mdu438.8.

27. Yang YH, Lin JK, Chen WS, et al. Comparison of cetuximab to bevacizumab as the first-line bio-chemotherapy for patients with metastatic colorectal cancer: superior progression-free survival is restricted to patients with measurable tumors and objective tumor response - a retrospective study. J Cancer Res Clin Oncol. 2014;140(11):1927-1936.

28. Hecht JR, Cohn A, Dakhil S, et al. SPIRITT: a randomized, multicenter, Phase II study of panitumumab with FOLFIRI and bevacizumab with FOLFIRI as second-line treatment in patients with unresectable wild type KRAS metastatic colorectal cancer. Clin Colorectal Cancer. 2015;14(2):72-80.

29. Heinemann V, Niedzwiecki D, Rachel V, et al. Outcomes for FOLFIRI plus bevacizumab (BEV) or cetuximab (CET) in patients previously treated with oxaliplatin-based adjuvant therapy: a combined analysis of data from FIRE-3 and CALGB 80405. J Clin Oncol. 2015;33: (Suppl; abstract 3585).

30. Douillard JY, Siena S, Cassidy J, et al. Final results from PRIME: randomized Phase III study of panitumumab with FOLFOX4 for firstline treatment of metastatic colorectal cancer. Ann Oncol. 2014;25(7): 1346-1355.

31. Saltz LB, Clarke S, Diaz-RE, et al. Bevacizumab in combination with oxaliplatin-based chemotherapy as first-line therapy in metastatic colorectal cancer: a randomized Phase III study. J Clin Oncol. 2008; 26(12):2013-2019.

32. Elez E, Argiles G, Tabernero J. First-line treatment of metastatic colorectal cancer: interpreting FIRE-3, PEAK, and CALGB/SWOG 80405. Curr Treat Options Oncol. 2015;16(11):52.

33. Stintzing S, Modest DP, von Weikersthal LF, et al. LBA11independent radiological evaluation of objective response, early tumor shrinkage, and depth of response in FIRE-3 (AIO KRK-0306) in the final RAS evaluable population. Ann Oncol. 2014;25(Suppl 4):mdu438.439.

34. Modest DP, Stintzing S, von Weikersthal LF, et al. Impact of subsequent therapies on outcome of the FIRE-3/AIO KRK0306 trial: First-line therapy with FOLFIRI plus cetuximab or bevacizumab in patients with KRAS wild-type tumors in metastatic colorectal cancer. J Clin Oncol. 2015;33(32):3718-3726. 
35. Ciardiello F, Bianco R, Caputo R, et al. Antitumor activity of ZD6474, a vascular endothelial growth factor receptor tyrosine kinase inhibitor, in human cancer cells with acquired resistance to antiepidermal growth factor receptor therapy. Clin Cancer Res. 2004;10(2):784-793.

36. Viloria-Petit A, Crombet T, Jothy S, et al. Acquired resistance to the antitumor effect of epidermal growth factor receptor-blocking antibodies in vivo: a role for altered tumor angiogenesis. Cancer Res. 2001; 61(13):5090-5101.

37. Maughan TS, Adams RA, Smith CG, et al. Addition of cetuximab to oxaliplatin-based first-line combination chemotherapy for treatment of advanced colorectal cancer: results of the randomised phase $3 \mathrm{MRC}$ COIN trial. Lancet. 2011;377(9783):2103-2114.

38. Feng QY, Wei Y, Chen JW, et al. Anti-EGFR and anti-VEGF agents: important targeted therapies of colorectal liver metastases. World $J$ Gastroenterol. 2014;20(15):4263-4275.

39. Stremitzer S, Sebio A, Stintzing S, Lenz HJ. Panitumumab safety for treating colorectal cancer. Expert Opin Drug Saf. 2014;13(6):843-851.

40. Berger MD, Lenz HJ. The safety of monoclonal antibodies for treatment of colorectal cancer. Expert Opin Drug Saf. 2016;15(6):799-808.

41. Bokemeyer C, Kohne CH, Ciardiello F, et al. Treatment outcome according to tumor RAS mutation status in OPUS study patients with metastatic colorectal cancer (mCRC) randomized to FOLFOX4 with/ without cetuximab. J Clin Oncol. 2014;32(5s):(Suppl; abstract 3505).

42. Ciardiello F, Lenz H, Kohne CH, et al. Treatment outcome according to tumor RAS mutation status in CRYSTAL study patients with metastatic colorectal cancer (mCRC) randomized to FOLFIRI with/without cetuximab. J Clin Oncol. 2014;32(5s):(Suppl; abstract 3506).
43. Laurent-Puig P, Cayre A, Manceau G, et al. Analysis of PTEN, BRAF, and EGFR status in determining benefit from cetuximab therapy in wild-type KRAS metastatic colon cancer. J Clin Oncol. 2009;27(35): 5924-5930.

44. Loupakis F, Ruzzo A, Cremolini C, et al. KRAS codon 61, 146 and BRAF mutations predict resistance to cetuximab plus irinotecan in KRAS codon 12 and 13 wild-type metastatic colorectal cancer. $\mathrm{Br} J$ Cancer. 2009;101(4):715-721.

45. Chan D, Pavlakis N, Price TJ, et al. Impact of chemotherapy partner on efficacy of targeted therapy in metastatic colorectal cancer (mCRC): a meta-analysis. J Clin Oncol. 2014;32(5s):(Suppl; abstract 3552).

46. Fakih MG. Metastatic colorectal cancer: current state and future directions. J Clin Oncol. 2015;33(16):1809-1824.

47. Giantonio BJ, Catalano PJ, Meropol NJ, et al. Bevacizumab in combination with oxaliplatin, fluorouracil, and leucovorin (FOLFOX4) for previously treated metastatic colorectal cancer: results from the Eastern Cooperative Oncology Group Study E3200. J Clin Oncol. 2007;25(12):1539-1544.

48. Peeters M, Price TJ, Cervantes A, et al. Randomized phase III study of panitumumab with fluorouracil, leucovorin, and irinotecan (FOLFIRI) compared with FOLFIRI alone as second-line treatment in patients with metastatic colorectal cancer. J Clin Oncol. 2010;28(31):4706-4713. 


\section{Supplementary materials}

Table SI Risk of bias of RCTs (the Jadad scale)

\begin{tabular}{|c|c|c|c|c|c|}
\hline Study & Randomization & Blinding & Withdraw and dropout & Jadad's score & Quality \\
\hline Heinemann et al' ${ }^{12}$ & 2 & I & 2 & 5 & High \\
\hline Schwartzberg et al ${ }^{14}$ & 2 & I & I & 4 & High \\
\hline Hecht et $\mathrm{al}^{28}$ & 2 & I & I & 4 & High \\
\hline
\end{tabular}

Notes: Randomization: randomization was described with appropriate method: 2 score; randomization was described without appropriate method: I score; no randomization: 0 score. Blinding: blinding was performed on all doctors and patients: 2 score blinding was partially performed on doctors and patients: I score; no blinding: 0 score. Withdraw and dropout: the reason of withdraw and dropout was described: I score; the reason of withdraw and dropout was not described: 0 score; Quality: high-quality trials should score $\geq 3$.

Abbreviation: RCTs, randomized controlled trials.

Table S2 Risk of bias of retrospective studies (NOS)

\begin{tabular}{|c|c|c|c|c|c|c|c|c|c|c|c|}
\hline \multirow[t]{2}{*}{ Study } & \multicolumn{4}{|c|}{ Selection } & \multicolumn{2}{|c|}{ Comparability } & \multicolumn{3}{|c|}{ Outcome } & \multirow[t]{2}{*}{ Total } & \multirow[t]{2}{*}{ Quality } \\
\hline & REC & SNEC & $\mathbf{A E}$ & DO & SC & AF & AO & FU & FUO & & \\
\hline Stremitzer et $\mathrm{al}^{25}$ & 1 & 0 & I & I & 0 & 0 & I & 1 & I & 6 & Moderate \\
\hline Yang et $\mathrm{al}^{27}$ & 1 & 0 & I & I & 0 & 0 & I & 1 & 1 & 6 & Moderate \\
\hline
\end{tabular}

Abbreviations: REC, representativeness of the exposed cohort; SNEC, selection of the nonexposed cohort; AE, ascertainment of exposure; DO, demonstration that outcome of interest was not present at start of study; SC, study controls for age and sex; AF, study controls for any additional factors; AO, assessment of outcome; FU, follow-up long enough for outcomes to occur; FUO, adequacy of follow-up of cohorts; NOS, Newcastle-Ottawa Scale.

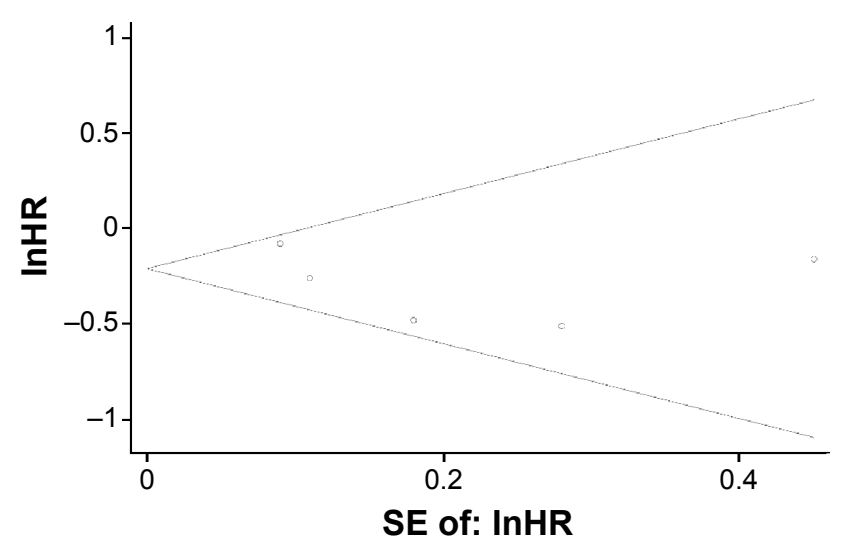

Figure SI Begg's funnel plot with pseudo $95 \%$ confidence limits on OS. Abbreviations: HR, hazard ratio; SE, standard error; OS, overall survival.

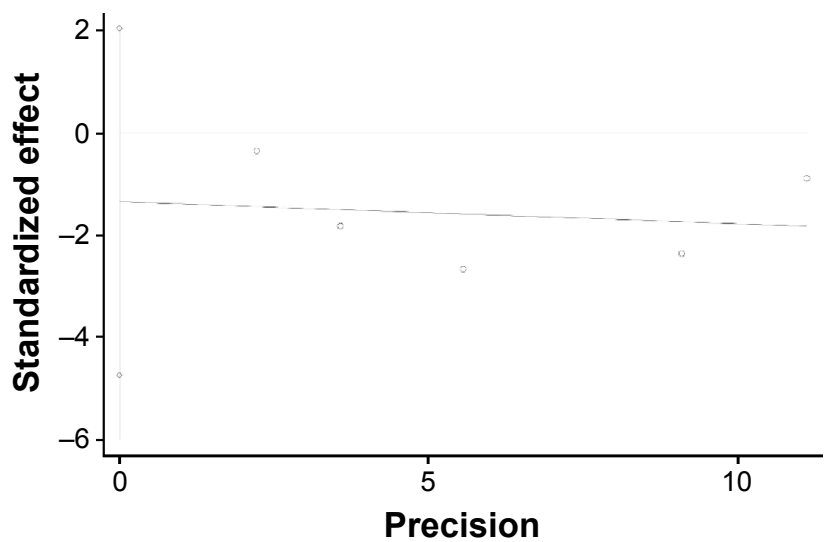

Figure S2 Egger's publication bias plot on OS

Abbreviation: OS, overall survival.

\section{Publish your work in this journal}

OncoTargets and Therapy is an international, peer-reviewed, open access journal focusing on the pathological basis of all cancers, potential targets for therapy and treatment protocols employed to improve the management of cancer patients. The journal also focuses on the impact of management programs and new therapeutic agents and protocols on
Dovepress

patient perspectives such as quality of life, adherence and satisfaction The manuscript management system is completely online and includes a very quick and fair peer-review system, which is all easy to use. Visit http://www.dovepress.com/testimonials.php to read real quotes from published authors. 